\title{
NOTES D'ACTUALITÉ
}

\section{Les suites de la pandémie devront être faites d'innovations territoriales}

\section{Yannick Hémond ${ }^{\mathrm{a}}$}

La pandémie de la COVID-19 a frappé la majorité des pays et a révélé notre manque de préparation à ce type d'aléa. Parmi les constats, nous n'avions pas la capacité dans le milieu de la santé pour traiter l'ensemble des personnes infectées, il manquait de l'équipement et l'approvisionnement n'était pas simple. Les réactions des gouvernements ont consisté à mettre en pause l'économie et à confiner les gens.

Ces mesures hors-normes ont permis de répondre à une situation hors-norme. L'objectif premier était d'assurer une capacité de traitement des personnes atteintes de la COVID-19 et d'éviter un débordement du système de santé. Or, le temps de confinement a fait ressortir plusieurs vulnérabilités sociales. Que ce soit dans les centres pour personnes âgées (résidences privées, centres d'hébergement et de soins de longue durée) ou chez les personnes vivant dans un contexte de pauvreté financière, la pandémie révèle de manière claire la défaillance de nos mesures pour soutenir les personnes vulnérables dans nos sociétés.

Les actions pour gérer la crise pandémique travers le monde révèlent donc les vulnérabilités systémiques que nous avons collectivement construites avec le temps. Plusieurs mesures mises en place au cours des dernières semaines à Montréal nous démontrent que notre rapport au territoire et, surtout, notre occupation du territoire devront être repensés. Que ce soit les corridors sanitaires, les rues piétonnisées, l'utilisation du vélo ou le transport collectif, notre façon de nous déplacer devra être améliorée. Il en est de même concernant le confinement régional. Les déplacements entre les régions font peu à peu place à une réouverture. Comment voyagerons-nous pendant l'été 2020?

Collectivement, nous devons nous préparer à vivre avec ces bouleversements, qui seront plus nombreux et qui se chevaucheront. La pandémie ne fait pas disparaitre les autres enjeux : elle les exacerbe autant qu'elle le fait avec les vulnérabilités. La solution se trouve inévitablement dans notre rapport au territoire, à l'environnement et à l'organisation sociale. Nous avons besoin d'identifier les facteurs de vulnérabilité non pas en fonction d'une pandémie, mais sous l'angle des innovations territoriales possibles dans le futur.

Nous commençons à peine à voir ce qu'il est possible de mettre en place pour parvenir à redéfinir notre rapport au territoire. Il faudra nous donner le temps de réfléchir à ces relations, si nous voulons trouver des solutions durables et acceptées par la population. La période qui s'en vient devra faciliter la mise en place d'innovations territoriales. Les solutions durables à long terme passeront entre autres par la mobilisation du milieu communautaire et des citoyens. Directement associée à la résilience urbaine, cette mobilisation offrira la capacité de traiter et de prendre en charge les plus vulnérables de nos sociétés.

a Professeur, département de géographie, Université du Québec à Montréal 
Cette période d'incertitude et d'inconnu doit nous permettre cette reconnexion nécessaire à l'environnement. Ce confinement nous aura permis de nous réapproprier les espaces communs d'une nouvelle manière et avec de nouvelles règles. Les gens ont pu redécouvrir leur quartier, les parcs urbains (avec une distanciation physique) et les commerces de proximité, qui s'adaptent pour accommoder leurs clients.

Au Québec, heureusement, la période des inondations printanières n'est pas venue exacerber les conséquences de la pandémie. Les autorités de santé publique et plusieurs municipalités sont d'ailleurs en réflexion concernant la gestion des périodes de canicule estivale en contexte de pandémie. Plusieurs îlots de chaleur sont présents dans nos grandes villes. L'indice de canopée y étant souvent faible, il y aura des défis pour prendre en charge les personnes vulnérables à la canicule, qui sont d'ailleurs les mêmes qui sont vulnérables à la pandémie.

Ces nouveaux défis pour les gestionnaires municipaux ne constituent qu'un aperçu de l'impact à long terme des changements climatiques sur le territoire. 